\title{
Folding, activity and import of steroidogenic acute regulatory protein into mitochondria changed by nicotine exposure
}

\author{
Mahuya Bose, Dilip Debnath, Yue Chen and Himangshu S Bose \\ Department of Physiology and Functional Genomics, University of Florida, Room M 552, Box 100274, 1600 SW Archer Road, Gainesville, Florida 32610 , USA \\ (Requests for offprints should be addressed to H S Bose; Email: hbose @ufl.edu)
}

\begin{abstract}
Nicotine, a pharmacologically active constituent of tobacco smoke, decreases sex steroid production and impairs reproductive function. The rate-limiting step in steroid hormone biosynthesis is the transport of substrate cholesterol from the outer to inner mitochondrial membrane by the steroidogenic acute regulatory protein (StAR). StAR is a $37 \mathrm{kDa}$ cytoplasmic phosphoprotein processed as a $32 \mathrm{kDa}$ intermediate to a mature $30 \mathrm{kDa}$ inactive mitochondrial protein. StAR's cholesterol transport capacity is proportional to its residency time at the outer mitochondrial membrane (OMM). Nonsteroidogenic COS-1 cells transfected with StAR/F2, steroidogenic MA-10 cells induced with cAMP or transfected with StAR or the isolated steroidogenic mitochondria preincubated with nicotine reduced StAR expression, import and activity. Mitochondria isolated from steroidogenic tissues or cells, pretreated with nicotine, also reduced the association of StAR with the OMM, but had no effect on the import of signal sequence substituted SCC/N-62StAR. The fluorescence emission maximum of StAR was unchanged with nicotine, but StAR's free energy of unfolding and the surface area $(m)$ increased in the presence of nicotine. Nicotine also blocked StAR from proteolysis with trypsin, suggesting that nicotine partially stabilised protein conformation by insertion into the molten globule conformation of StAR.
\end{abstract}

Journal of Molecular Endocrinology (2007) 39, 67-79

\section{Introduction}

Smoking causes $\sim 4$ million deaths in the United States every year. Nicotine, one of the main components of cigarette smoke, is delivered through smoking and is not only a potent cause of lung cancer but also causes low birth rate, preterm delivery and abortion in women addicted to smoking. Nicotine causes menstrual irregularities and pregnancy complications, decreases fertility in women (Weisberg 1985), inhibits spermatogenesis and causes decreased steroidogenesis (Aydos et al. 2001, Mlynarcikova et al. 2005). From the study of laboratory animals, nicotine affects normal functioning of accessory reproductive organs in male albino rats (Patil et al. 1999). Although nicotine's mechanism of action is not clearly understood, it inhibits the release of pituitary follicle-stimulating hormone and luteinizing hormone through its action on the hypothalamohypophyseal axis (Blake et al. 1972, Blake 1974, 1978). Cigarette smoke contains an average of $0.5-1.6 \mathrm{mg}$ nicotine per cigarette (Calafat et al. 2004, Shihadeh \& Saleh 2005), which was found to be $0.01 \mu \mathrm{g} / \mathrm{ml}$ $(0.022 \mu \mathrm{M})$ in the blood stream and $1 \mu \mathrm{g} / \mathrm{ml}$ or $2 \cdot 2 \mu \mathrm{M}$ in the tissues of smokers (Hakki et al. 2001). Nicotine dependence results from binding of nicotine to acetylcholine receptors in the central nervous system (Tapper et al. 2004), thus it influences the central nervous system and also increases the oxidative stress of mitochondria, which in turn affects normal respiratory function of the mitochondrion, a key organelle in the first step of steroid hormone biosynthesis.

In an acute response, steroid hormones are synthesised and released within minutes of tropic hormonal stimulation. Steroidogenic tissues do not store presynthesised steroids but respond rapidly to increase the conversion of cholesterol to steroid hormones under acute conditions (Miller 1988). The substrate cholesterol is insoluble in water and is transported into the mitochondria by an unknown mechanism. The ratelimiting step is the transport of cholesterol from the outer to inner mitochondrial membrane and is fostered by a cytoplasmic phosphoprotein called the steroidogenic acute regulatory protein or StAR (Stocco \& Clark 1996). StAR is a $37 \mathrm{kDa}$ phosphorylated 'active preprotein', and is transported into mitochondria as a $30 \mathrm{kDa}$ mature protein (Stocco \& Clark 1996). Mutant StAR cannot transport cholesterol, resulting in an inborn error in metabolism, called congenital lipoid adrenal hyperplasia (lipoid CAH), in which fetuses die shortly after birth due to a salt-loosing crisis (Lin et al. 1995, Bose et al. 1996, 2000, Nakae et al. 1997). StAR is a 285 amino acid protein, but deletion of the first 62 amino acids retains complete activity when compared with full-length StAR in the transfected cells or when biosynthetic N-62 StAR or cell-free synthesised (CFS) StAR is incubated with mitochondria isolated from 
MA-10 cells. On the contrary, carboxy-terminal deletion of only 10 amino acids reduce StAR activity by $50 \%$ and deletion of 28 amino acids completely abolishes the same (Lin et al. 1995, Sugawara et al. 1995, Arakane et al. 1996). Biochemical and structural studies have shown that the StAR amino acid substitution mutations that cause lipoid CAH have a gross error in folding (Bose et al. 1998). Therefore, the movement of cholesterol into mitochondria is dependent on two factors: first, the expression of properly folded StAR, and second, the interaction of StAR with mitochondrial membranes. Thus, any hindrance of StAR association with the OMM will reduce cholesterol transport into the mitochondria and will reduce pregnenolone synthesis. Environmental factors, such as exposure to tobacco smoke which contains nicotine, may reduce the interaction of StAR with the OMM, resulting in reduced transport of the substrate cholesterol and finally reduced pregnenolone. In this article, we report that the presence of nicotine inhibits StAR processing and cholesterol transport into mitochondria, thereby decreasing pregnenelone synthesis. We also provide evidence that StAR import occurs in a unique fashion through the OMM and that nicotine changes StAR-specific mitochondrial import processing at the OMM.

\section{Materials and methods}

\section{Cell culture and transfection}

COS-1 cells (monkey kidney) were grown in $10 \mathrm{~cm}$ plates in Dulbecco's modified Eagle medium supplemented with $10 \%$ foetal bovine serum and $1 \times$ penicillinstreptomycin at $37^{\circ} \mathrm{C}$ in a humidified incubator with $5 \% \mathrm{CO}_{2}$. Cells were plated at a density of $1 \times 10^{6}$ in six-well plates $24 \mathrm{~h}$ prior to transfection, and co-transfected with $1.0 \mu \mathrm{g}$ StAR and F2 (P450scc-Adrenodoxin reductaseadrenodoxin; Harikrishna et al. 1993) using lipofectamine (Invitrogen). The cells were washed with serum-free media $12 \mathrm{~h}$ after transfections, and supplemented with media containing appropriate antibiotics, $10 \%$ serum and 1 pmol trilostane. The media was collected after $48 \mathrm{~h}$ and the accumulated pregnenolone was measured by RIA (RIA kit, MP Biomedicals, Irvine, CA, USA). To expose cells with nicotine, we incubated either COS-1 or MA-10 (mouse leydig) cells in serum-free media containing $0 \cdot 1$, $0 \cdot 5,1 \cdot 0,2 \cdot 5,5 \cdot 0$ and $10 \mathrm{mM}$ nicotine for $6 \mathrm{~h}$, then removed the nicotine and allowed the cells to recover for $2 \mathrm{~h}$ in complete growth media. After $2 \mathrm{~h}$, both nicotine-treated and -untreated cells were co-transfected with plasmids containing StAR and F2 vectors, and accumulated pregnenolone was measured as described above. For PCR amplification of the nicotine-exposed cells, RNA was isolated using TRIZOL reagent (Invitrogen), and reverse transcribed with Superscript II with 'oligodT' primer at
$42{ }^{\circ} \mathrm{C}$. Two microlitres of cDNA were amplified by $5^{\prime}-$ AGCTATGGAAGAGACTCTCTA- $3^{\prime}$ sense and $5^{\prime}$-AGCTAAGCTTCAACACCTGGCT- $3^{\prime}$ anti-sense primer for 25 cycles at $94^{\circ} \mathrm{C}$ for $1 \mathrm{~min}, 72^{\circ} \mathrm{C}$ for $2 \mathrm{~min}$ and $55^{\circ} \mathrm{C}$ for $1 \mathrm{~min}$. An aliquot of $10 \mu \mathrm{l}$ amplified DNA was analysed in a $1.6 \%$ agarose gel stained with ethidium bromide.

\section{Western blot}

The protein concentration was determined using BioRad's dye reagent (Bio-Rad). Protein samples $(12.5 \mu \mathrm{g})$ from transfected cells were electrophoresed in $15 \%$ SDS-PAGE, electroblotted to a polyvinylidene flouride (PVDF) membrane and processed for western blotting with N-62 StAR (Bose et al. 1999), cytochrome P450 side chain cleavage enzyme (Hsu et al. 2006) and adrenodoxin reductase, antibodies independently (Brentano et al. 1992). As a control for StAR, we used Escherichia coli expressed N-62 StAR and CFS P450scc as a control for F2. The band intensity was quantitated by digitising with the 'Unscan-it' program (Silk Scientific Corporation, Orem, UT, USA).

\section{Isolation and purification of mitochondria}

Protein import and competition experiments require large quantities of mitochondria; since milligram quantities of mitochondria can be isolated from a pair of adrenals, we preferred to use mitochondria from sheep adrenal tissues for all import and direct bioactivity measurements. The sheep adrenals, from control groups, were available from the laboratory of Dr Charles Wood, University of Florida, immediately after killing. To isolate the mitochondria from adrenal glands, the tissues were transferred to mitochondria isolation buffer (250 mM sucrose, $10 \mathrm{mM}$ HEPES ( $\mathrm{pH} 7 \cdot 4), 1 \mathrm{mM}$ EGTA) immediately after killing and chopped into small pieces on ice. The cells or the tissue fractions were homogenised in a hand-held all-glass Dounce homogeniser with ten gentle up and down strokes, and the cell debris was removed by spinning at $3500 \mathrm{~g}$ for $10 \mathrm{~min}$. The supernatant containing the mitochondrial fraction was centrifuged at $10000 \mathrm{~g}$ for $10 \mathrm{~min}$, and the pellet was washed with the mitochondrial isolation buffer, resuspended in an energy regeneration buffer $(125 \mathrm{mM}$ sucrose, $80 \mathrm{mM} \mathrm{KCl}, 5 \mathrm{mM} \mathrm{MgCl} 2,10 \mathrm{mM} \mathrm{NaH}{ }_{2} \mathrm{PO}_{4}$, $10 \mathrm{mM}$ isocitrate, $1.0 \mathrm{mM}$ ATP, $1.0 \mathrm{mM}$ NADP, $0 \cdot 1 \mathrm{mM}$ ADP and $25 \mathrm{mM}$ HEPES (pH 7.4) ) and stored at either $-86^{\circ} \mathrm{C}$ or in liquid nitrogen.

\section{In vitro steroidogenic activity with biosynthetic N-62 StAR}

To isolate the biologically active form of N-62 StAR from E. coli cytosol, the bacterial pellet was sonicated in 
a glycerol buffer (10 mM HEPES (pH 7.4), 10\% glycerol and $1 \mathrm{mM}$ phenylmethylsulphonyl fluoride (PMSF)) on ice for $10 \mathrm{~min}$. The collected supernatant was centrifuged at $18000 \mathrm{~g}$ for $10 \mathrm{~min}$, passed through a Ni-NTA superpose column, washed with glycerol buffer and eluted with the same buffer containing $100 \mathrm{mM}$ imidazole. The final purification was done by passing through a gel filtration column equipped with a fast protein liquid chromatography (FPLC) system (Pharmacia, AKTA). To determine the StAR activity, we incubated $1 \mu \mathrm{g}$ biosynthetic StAR with $20 \mu \mathrm{g}$ sheep adrenal mitochondria in an energy regeneration buffer (Bose et al. 2002) for $1 \mathrm{~h}$ at $37^{\circ} \mathrm{C}$ and measured the pregnenolone synthesis by RIA.

\section{Protein import}

$\left[{ }^{35} \mathrm{~S}\right]$-Met labelled StAR and SCC/StAR were synthesised in a cell-free system (CFS) using a transcription/translation (TNT) coupled reticulocyte lysate systems (Promega). Ribosomes and associated incompletely translated polypeptide chains were removed by centrifugation at $150000 \mathrm{~g}$ for $15 \mathrm{~min}$ at $4{ }^{\circ} \mathrm{C}$ (Schwartz \& Matouschek 1999). For all protein import experiments, $100 \mu \mathrm{g}$ isolated mitochondria was incubated in a $26{ }^{\circ} \mathrm{C}$ water bath with ${ }^{35} \mathrm{~S}$-StAR or SCC/ StAR to a final volume of $100 \mu$, and terminated by the addition of $1 \mathrm{mM} \mathrm{mCCCP}$ and an equal volume of boiling $2 \times$ SDS-sample buffer. The import reactions were analysed by electrophoresing through SDS-PAGE, fixed in methanol/acetic acid (40:10), dried and exposed to a phosphorimager screen.

For the isolation of the StAR-OMM complex, $\left[{ }^{35} \mathrm{~S}\right] \mathrm{StAR}$ and P450scc were imported into isolated mitochondria, extracted with digitonin buffer $(1 \%$ $(\mathrm{w} / \mathrm{v})$ digitonin, $20 \mathrm{mM}$ Tris-HCl $(\mathrm{pH} 7 \cdot 4), 0 \cdot 1 \mathrm{mM}$ EDTA, $50 \mathrm{mM} \mathrm{NaCl}, 10 \%$ (w/v) glycerol, $1 \mathrm{mM}$ PMSF) for $15 \mathrm{~min}$ on ice and subjected to gradient blue native (BN)-PAGE (Schagger \& von Jagow 1993, Schagger et al. 1994) at $4{ }^{\circ} \mathrm{C}$ and $100 \mathrm{~V}$ overnight. An aliquot of $100 \mu \mathrm{l}$ digitonin lysate was applied on a $30-10 \%$ sucrose density gradient, centrifuged for $4 \mathrm{~h}$ at $4{ }^{\circ} \mathrm{C}$ at $286000 \mathrm{~g}$ in a Beckman ultracentrifuge, aliquoted into equal fractions and analysed by SDS-PAGE.

\section{Cigarette smoke condensate (CSC)}

The CSC was prepared at the University of Kentucky using Reference Cigarettes, 1R4F (9 mg tar and 0.8 mg nicotine/cig; Pillsbury \& Bright 1972). Briefly, the smoke particulates were collected on a Cambridge filter pad from cigarettes smoked under the standard Federal Trade Commission protocol $(35 \mathrm{ml}$ puff volume of a $2 \mathrm{~s}$ duration; Pillsbury \& Bright 1972) and dissolved in dimethylsulfoxide (DMSO) at $40 \mathrm{mg} / \mathrm{ml}$. Control cells were treated with a medium containing an equivalent amount of DMSO.

\section{Fluorescence spectroscopy}

For fluorescence spectroscopy, Tryptophan (Trp) was excited at $295 \mathrm{~nm}$ and the spectrum was recorded at $0.2 \mathrm{~nm}$ intervals from 310 to $450 \mathrm{~nm}$ at $25^{\circ} \mathrm{C}$. The buffer $\left(20 \mathrm{mM} \mathrm{NaH}{ }_{2} \mathrm{PO}_{4}, 50 \mathrm{mM} \mathrm{NaCl}, \mathrm{pH} 7 \cdot 4\right)$ and protein spectra were recorded under identical conditions. Emission maxima and intensity were plotted as a function of increasing amounts of nicotine. Because of the high photon counts from the N-62 StAR, we employed only $10 \mathrm{ng}$ protein throughout the experiment. For unfolding studies, N-62 StAR was incubated with $0 \cdot 01,0 \cdot 1,1 \cdot 0,5$ and $10 \mathrm{mM}$ nicotine for $2 \mathrm{~h}$ and then melted by incubating with $8.0 \mathrm{M}$ urea overnight at room temperature. Wavelength maxima of each fluorescence spectrum were determined after subtraction of buffer blank, plotted as a function of urea concentration and the free energy $(\Delta G)$ of unfolding was calculated by following the linear extrapolation (Pace 1986).

\section{Results}

\section{Nicotine reduces pregnenolone synthesis by inhibit- ing StAR expression and processing}

To study the effect of nicotine on pregnenolone synthesis, we incubated COS-1 cells with different concentrations of nicotine prior to transfection with StAR and F2 vector. Nonsteroidogenic COS-1 cells do not express the P450scc system, so we co-transfected with F2 vector which expresses the complete P450scc system as a single fusion protein (P450scc-adrenodoxin reducatse-adrenodoxin; Harikrishna et al. 1993). As a positive control, we transfected the COS-1 cells with F2 and water soluble 22R-hydroxycholesterol, which bypasses the action of StAR and shows the maximum capacity of a cell to synthesise steroid hormones. Nicotine showed no effect on pregenolone synthesis up to $1.0 \mathrm{mM}$ concentration, but increasing the nicotine concentration to $2.5 \mathrm{mM}$ decreased pregnenolone from 480 to $320 \mathrm{ng} / \mathrm{ml}$, a loss of $33 \%$ in comparison to the untreated cells (Fig. 1A). At $5 \mathrm{mM}$ nicotine, pregnenolone was reduced to $220 \mathrm{ng} / \mathrm{ml}$ and at $10 \mathrm{mM}$ the activity was decreased to the level of buffer control, suggesting a strong inhibition of pregnenolone synthesis at higher concentrations of nicotine. StAR expression was unchanged with $<1 \mathrm{mM}$ nicotine (Fig. 1B), but with higher concentrations and especially at $2.5 \mathrm{mM}$ concentrations of nicotine, the 37 and $32 \mathrm{kDa}$ StAR expressions (Fig. 1C) were completely inhibited. Expression of F2, which is a $120 \mathrm{kDa}$ protein, monitored by western blotting with anti-P450scc (Fig. 1D) and 
A

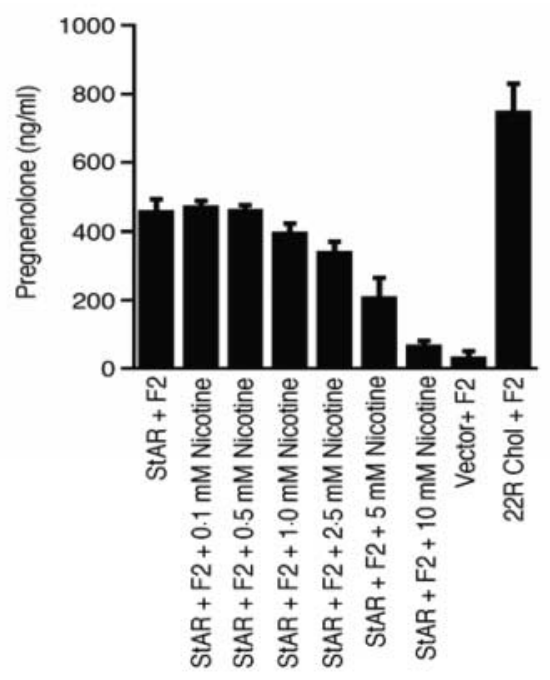

B

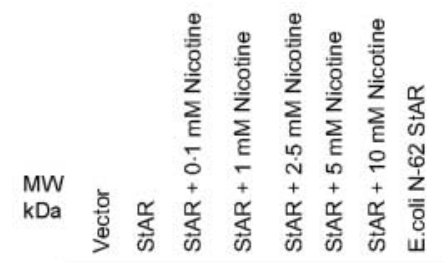

C
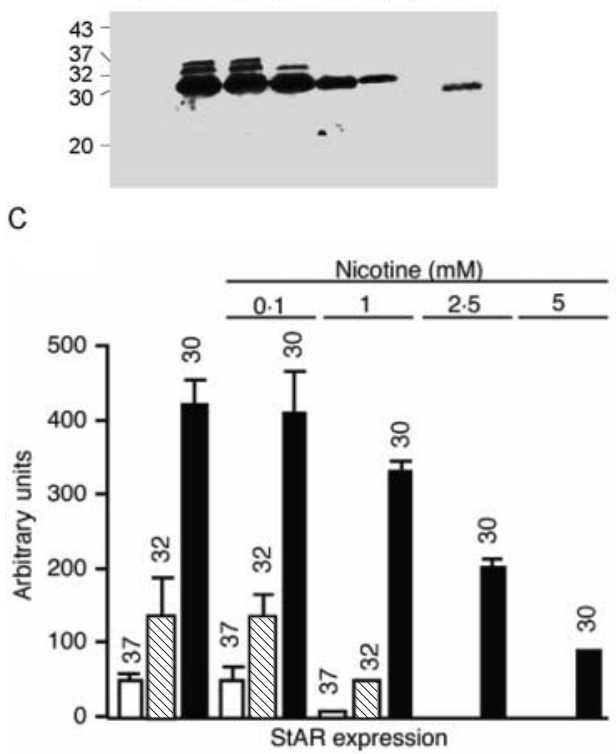

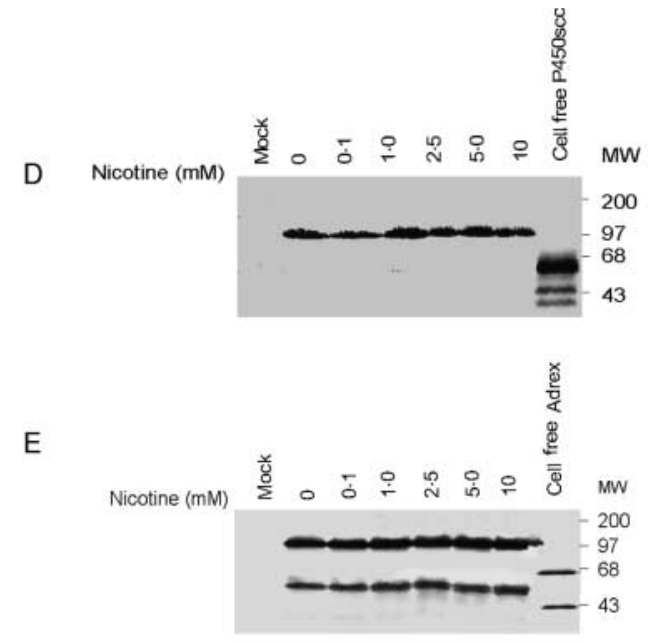

F

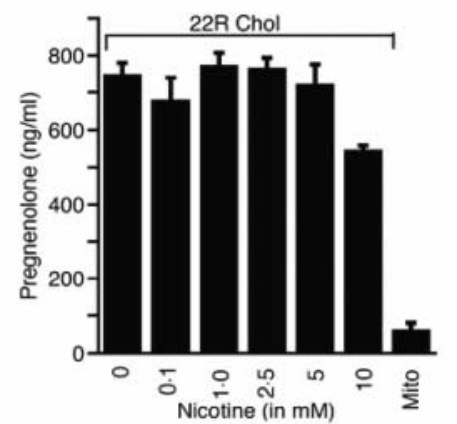

G

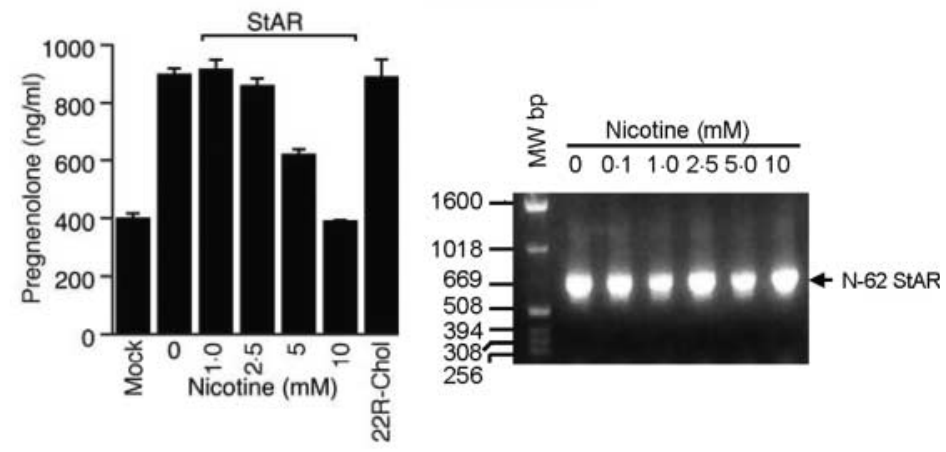

$H$

$\sum_{\Sigma}^{\circ}$

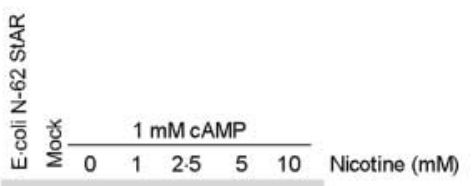

$37-$
$32-$
$30-$

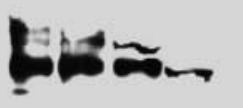

Figure $1 \mathrm{Effect}$ of nicotine on pregnenolone synthesis. (A) COS-1 cells were preincubated with $0 \cdot 1,0 \cdot 5,1 \cdot 0,2 \cdot 5,5 \cdot 0$ and $10 \mathrm{mM}$ of nicotine and co-transfected with StAR/F2. (B) Western blot with StAR antibody of the cells in (A). (C) Intensity of expression of StAR proteins, where $(\mathbb{\square})$ is $30 \mathrm{kDa}$, (四) is $32 \mathrm{kDa}$ and $(\square)$ is $37 \mathrm{kDa}$ StAR. (D and E) Western blot of the cells (A) with cytochrome P450scc and adrenodoxin reductase antibodies. Last lane, in (D), is the cell-free synthesised P450scc acting as positive control. (F) COS-1 cells preincubated with $0 \cdot 1,1 \cdot 0,2 \cdot 5,5 \cdot 0$ and $10 \mathrm{mM}$ nicotine, were transfected with F2 and incubated with $22 R-$ hydroxycholesterol. (G) Direct measurement of bioactivity from the mitochondria isolated from MA-10 cells preincubated with $0 \cdot 1,1 \cdot 0$, 
anti-adrenodoxin reductase (Fig. 1E) antibodies independently. The lower $45 \mathrm{kDa}$ band (Fig. 1E) with adrenodoxin reductase antibody may be due to a leaky expression of adrenodoxin reductase. This band is shorter than the full-length adrenodoxin reductaseexpressed band. In the F2 vector, adrenodoxin reductase is fused with the carboxy terminus of P450scc sequence without its $\mathrm{N}$-terminal amino acids, suggesting a possible reason for shorter expression than the full-length adrenodoxin reductase (Harikrishna et al. 1993). The pregnenolone synthesising capacity was determined by co-transfecting COS-1 cells with StAR and F2 vector, where the expression of F2 and StAR are under the control of the cytomegalovirus (CMV) promoter. To determine if nicotine affected the mitochondrial electron transport system, we measured pregnenolone synthesis with the F2-transfected COS-1 cells, incubated with 22Rhydroxycholesterol and different concentrations of nicotine. Only $10 \mathrm{mM}$ nicotine reduced the pregnenolone synthesis from 820 to $390 \mathrm{ng} / \mathrm{ml}$ with no effects observed at any lower concentrations (Fig. 1F). Similar results were observed when we transfected MA-10 cells with StAR in the presence of nicotine (Fig. 1G). The effect of nicotine depends on the mode of delivery (Benowitz 1996), and the in vitro experiments shows reduced pregnenolone synthesis only with higher concentrations of nicotine (Sarasin et al. 2003). Because of the faster hydrolysis of nicotine in the tissue, an accurate determination of nicotine concentration is not possible (Sarasin et al. 2003). The induction of MA-10 cells with cAMP in presence of nicotine, decreased StAR expression in a similar fashion as observed for COS-1 transfected with StAR and F2 (Fig. 1H). However, StAR mRNA expression remained unchanged, as determined by RT-PCR of MA10 cells RNA (Fig. 1G, right panel), indicating that nicotine directly inhibited StAR expression and processing into mitochondria without affecting the gene expression.

\section{Nicotine directly blocks cholesterol transport into mitochondria}

StAR's activity is directly proportional to its residency time at the OMM. We directly measured pregnenolone synthesis by incubating biosynthetic N-62 StAR with the mitochondria isolated from MA-10 cells and sheep adrenal tissues. The activity, as measured by the level of pregnenolone synthesis, remained at the similar level between the two sources of mitochondria (Fig. 2A) and showed no activity when incubated with heat-inactivated StAR, validating our mitochondrial preparation.
To understand nicotine-induced inhibition of cholesterol transport, we incubated sheep adrenal mitochondria with the biosynthetic N-62 StAR in the presence of $0 \cdot 1-10 \mathrm{mM}$ nicotine, and measured pregnenolone synthesis. Mitochondria incubated with the biosynthetic N-62 StAR synthesised $600 \mathrm{ng} / \mathrm{ml}$ pregenenolone (Fig. 2B), which was ninefold more than the mitochondria or buffer control. The pregnenolone level was reduced to $285 \mathrm{ng} / \mathrm{ml}$ with $2.5 \mathrm{mM}$ nicotine indicating that direct incubation of nicotine inhibited $47 \%$ of pregnenolone synthesis, which was significantly greater than the transfected cells. Increasing the nicotine concentration to $5.0 \mathrm{mM}$ reduced the pregnenolone to $75 \mathrm{ng} / \mathrm{ml}$, and with $10 \mathrm{mM}$ nicotine the pregnenolone synthesis was reduced to the level of buffer, suggesting that nicotine completely inhibited pregnenolone synthesis at higher concentrations. Incubation of biosynthetic N-62 StAR with the mitochondria isolated from MA-10 cells preexposed to nicotine also showed reduced pregnenolone (Fig. 2C). However, the pregnenolone synthesis was unaffected in presence of $10 \mathrm{mM}$ cotinine, a primary metabolite of nicotine demonstrating that indeed nicotine entered into the cell (Fig. 2C).

A direct incubation of nicotine may affect the mitochondrial electron transport system as nicotine depolarises the mitochondrial architecture (Banzet et al. 1999). Therefore, we determined the concentrationdependent effect of nicotine by incubating isolated adrenal mitochondria with 22R-hydroxycholesterol. The activity in the absence of nicotine was $780 \mathrm{ng} / \mathrm{ml}$, and remained unchanged up to $5 \mathrm{mM}$ (Fig. 2D) but decreased to $550 \mathrm{ng} /$ $\mathrm{ml}$ (Fig. 2D) at $10 \mathrm{mM}$ concentration. On western blotting, the expression of P450scc (61 kDa; Fig. 2E) and adrenodoxin reductase ( $54 \mathrm{kDa}$; Fig. $2 \mathrm{~F}$ ) remain unchanged. One shorter band of $55 \mathrm{kDa}$ with the P450scc antibody (Fig. 2E) is the signal sequence cleaved P450scc, and two smaller bands with the adrenodoxin antibody (Fig. 2F) are due to two-step cleavage of adrenodoxin reductase (Brentano $\mathrm{et} \mathrm{al}$. 1992), confirming that at lower concentrations nicotine have no effect on the mitochondrial electron transport system. The partial disappearance of the shorter band with the increase in nicotine concentration may be due to partial inhibition of the cleavage of the N-terminal signal sequence of adrenodoxin reductase.

\section{Nicotine inhibits StAR import}

To determine the effect of nicotine on StAR import through the OMM, we first characterised StAR import into isolated mitochondria by measuring the cleavage of

2.5, 5.0 and $10 \mathrm{mM}$ nicotine and transfected with StAR (left-hand panel). RT-PCR of the RNA from MA-10 cells incubated with $0 \cdot 1$, 1 , 2.5, 5 and $10 \mathrm{mM}$ nicotine (right-hand panel). (H) Western blot with StAR antibody of the cAMP induced MA-10 cell incubated with indicated concentrations of nicotine. Data in (A, C, F and G) (left panel) are the means ( \pm s.E.M.) of three separate experiments, each performed in triplicate. 
A

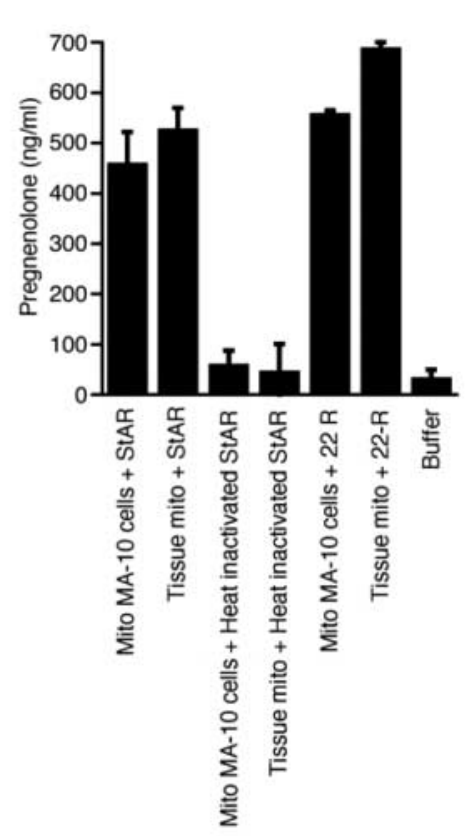

B

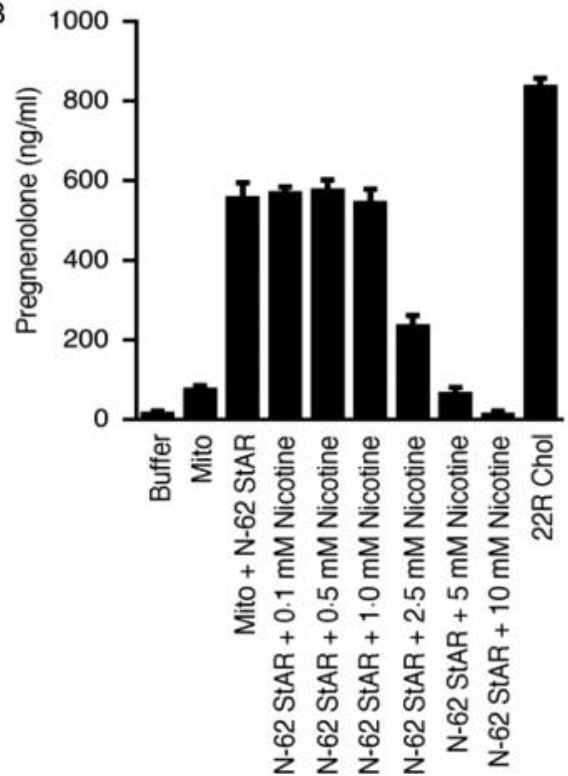

C
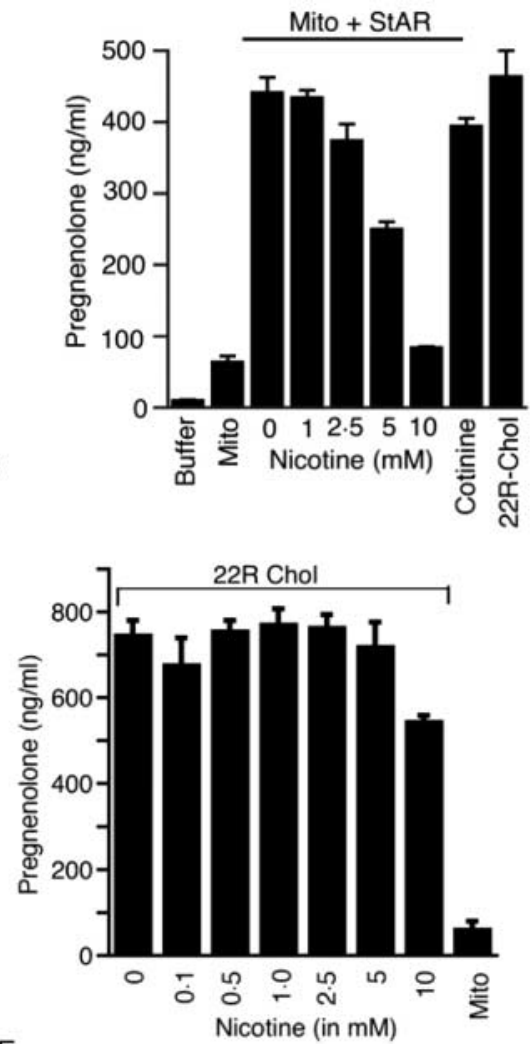

$\mathrm{E}$

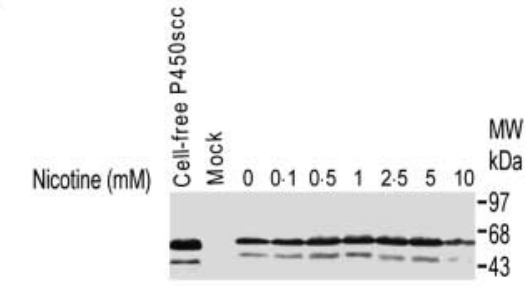

$\mathrm{F}$

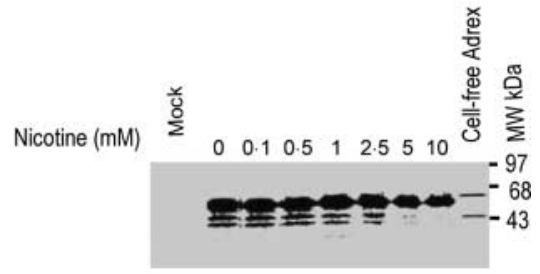

Figure 2 (A) Direct measurement of pregnenolone synthesis with the mitochondria isolated from MA-10 cells and adrenal tissues incubated with biosynthetic $\mathrm{N}-62$ StAR before and after heat inactivation. (B) Effect of nicotine on the isolated mitochondria. Mitochondria were incubated with the biosynthetic $\mathrm{N}-62$ StAR and $0 \cdot 1,0 \cdot 5,1 \cdot 0,2 \cdot 5,5 \cdot 0$ and $10 \mathrm{mM}$ of nicotine. (C) Direct measurement of bioactivity with the mitochondria isolated from MA-10 cells. An aliquot of $4.0 \mu \mathrm{g}$ mitochondria isolated from MA-10 cells incubated 1.0, 2.5, $5 \cdot 0$ and $10 \mathrm{mM}$ nicotine. One aliquot was also incubated with $10 \mathrm{mM}$ cotinine. (D) Incubation of 22R-hydroxylcholesterol with the mitochondria isolated from adrenal tissues in the presence of $0 \cdot 1,0 \cdot 5,1 \cdot 0,2 \cdot 5,5$ and $10 \mathrm{mM}$ nicotine. ( $E$ and $F$ ) Western blot with cytochrome P450scc and adrenodoxin reductase antibodies independent of the mitochondria exposed to the indicated concentrations of nicotine. Data in (A-D) are the mean ( \pm S.E.M.) of three separate independent experiments, each performed in triplicate. 
37 to $30 \mathrm{kDa}$ StAR. We also determined the membrane association of StAR by $\mathrm{Na}_{2} \mathrm{CO}_{3}$ extraction, as $\mathrm{Na}_{2} \mathrm{CO}_{3}$ breaks the protein-protein interaction ( $\mathrm{Li} \&$ Shore 1992), but not the lipid-protein interaction. Further, we compared StAR import efficiency with SCC/N-62 StAR by substituting its leader sequence with a mitochondrial matrix-targeting protein, P450scc. Import of ${ }^{35} \mathrm{~S}$-StAR and SCC/N-62 StAR onto the isolated mitochondria for $2 \mathrm{~h}$ (Fig. 3A) followed by extraction with sodium carbonate showed that StAR and SCC/N-62 StAR remained with the pellet fraction, suggesting that after import StAR and SCC/N-62 StAR were integrated into the membrane. However, the import efficiency of SCC/StAR is higher than StAR as determined by the intensity of the lower $26 \mathrm{kDa}$ band (Fig. 3B). Phosphorimager quantitation of the cleaved bands showed that the SCC/StAR was imported fivefold faster than StAR (Fig. 3C), demonstrating that protein import was kinetically controlled.

To identify the effect of nicotine on StAR import, we incubated the isolated mitochondria with $0 \cdot 1-10 \mathrm{mM}$ nicotine, and imported ${ }^{35} \mathrm{~S}-\mathrm{StAR}$ and ${ }^{35} \mathrm{~S}-\mathrm{SCC} / \mathrm{StAR}$. Nicotine concentrations up to $1 \mathrm{mM}$ had no effect on StAR import, but at higher concentrations the intensity of the $30 \mathrm{kDa}$ band was reduced (Fig. 3D). However, at any concentration of nicotine the import of SCC/StAR was unaffected. Incubation of additional fresh $100 \mu \mathrm{g}$ mitochondria to the mitochondria pre-exposed with nicotine ( 5 or $10 \mathrm{mM}$ ) restored StAR import (Fig. 3E), suggesting that nicotine indeed specifically inhibits StAR import. This also demonstrates that nicotine possibly blocked the StAR-specific mitochondrial import process, and that increasing the amount of mitochondria increased the free sites required for StAR import. To confirm this observation, we preincubated the isolated mitochondria with nicotine for $30 \mathrm{~min}$, reisolated, washed and then incubated with ${ }^{35} \mathrm{~S}-\mathrm{StAR}$ at $26^{\circ} \mathrm{C}$ for $2 \mathrm{~h}$ (Fig. 3F). Measurement of import efficiency (3G) showed that the $5 \mathrm{mM}$ or higher concentrations of nicotine inhibited StAR import, suggesting that temporary incubation of nicotine also inhibited the StAR-specific mitochondrial import process, but had no effect on the import of SCC/ StAR (3F, bottom panel).

\section{Effect of CSC on StAR activity and import}

Nicotine is delivered to the lungs and blood stream as a complex of several components during cigarette smoking. To determine the effect of the nicotine present in cigarette smoke, we incubated COS-1 cells with CSC as an index to cigarette smoke exposure and transfected them with StAR and F2 vector. Incubation of StAR/F2-transfected COS-1 cells with $0 \cdot 1 \mu \mathrm{g} / \mathrm{ml}$ of CSC had no effect, but $1 \mu \mathrm{g} / \mathrm{ml} \mathrm{CSC}$, which is equivalent to $70 \mathrm{ng}$ nicotine (RJ Reynolds Tobacco Co. 1988), reduced pregnenolone synthesis from 520 to $350 \mathrm{ng} / \mathrm{ml}(\sim 33 \%$ less $)$, and $5 \mu \mathrm{g} / \mathrm{ml}$ CSC reduced pregnenolone synthesis to the level of nontransfected cells (Fig. 4A). The tenfold increase in effect of CSC when compared with nicotine alone is due to the difference in delivery method of nicotine to the cells (Benowitz 1996). Western blotting of the transfected cells with StAR antiserum showed that $1 \mu \mathrm{g} / \mathrm{ml}$ CSC reduced the expression of $37 \mathrm{kDa}$ StAR by $75 \%$ and also reduced the expression of $30 \mathrm{kDa}$ StAR by $40 \%$. At higher concentrations of CSC the expression of $37 \mathrm{kDa}$ StAR was completely ablated, suggesting that the decrease in pregnenolone synthesis was due to the absence of $37 \mathrm{kDa}$ StAR (Fig. 4B and C). Nicotine binds directly with the mitochondrial membrane (Xie et al. 2005), and thus StAR may not have a chance to fold properly to bind with cholesterol and foster it to mitochondria. As a result, StAR was directly imported into mitochondria as a mature $30 \mathrm{kDa}$ protein without any increase in pregnenolone synthesis.

\section{Interaction of StAR with OMM is inhibited by nicotine}

To identify whether the presence of nicotine changes StAR's stability and its interaction with the OMM, we briefly imported full-length ${ }^{35} \mathrm{~S}$-StAR and P450scc into the mitochondria isolated from sheep adrenals, extracted with digitonin and electrophoresed on a 3-20\% BN-PAGE (BN Gel electrophoresis; Schagger \& von Jagow 1993, Schagger et al. 1994). We found only one complex with full-length StAR (Fig. 5A). To determine the inhibitory effect of nicotine we incubated the mitochondria in the presence and absence of nicotine, imported ${ }^{35} \mathrm{~S}-\mathrm{StAR}$, extracted with digitonin (Wiedemann et al. 2003) and fractionated the complex(es) by sucrose density gradient ultracentrifugation. Analysis of each fraction on SDS-PAGE (Fig. 5B and $\mathrm{C}$ ) showed two different distribution patterns of StAR complex: one with imported $30 \mathrm{kDa}$ StAR (bottom band) and one with unimported $37 \mathrm{kDa}$ StAR (top band). The nicotine-treated and -untreated mitochondria (Fig. 5B and $\mathrm{C}$ ) had a similar pattern of density distribution up to reaction number 7 (Fig. 5B), and the pattern changed from fraction 8 onwards with a reduction in intensity of the unimported $37 \mathrm{kDa}$ StAR complex, indicating that nicotine blocked StAR's interaction with the OMM.

\section{Binding of StAR with nicotine}

Protein folding is a dynamic process and folding changes upon binding or interaction with another protein. To understand whether a reduction in StAR expression is associated with its binding to nicotine, we used fluorescence spectroscopy probing with the Trp residues. Depending on the polarity of the Trp microenvironment, the fluorescence emission 


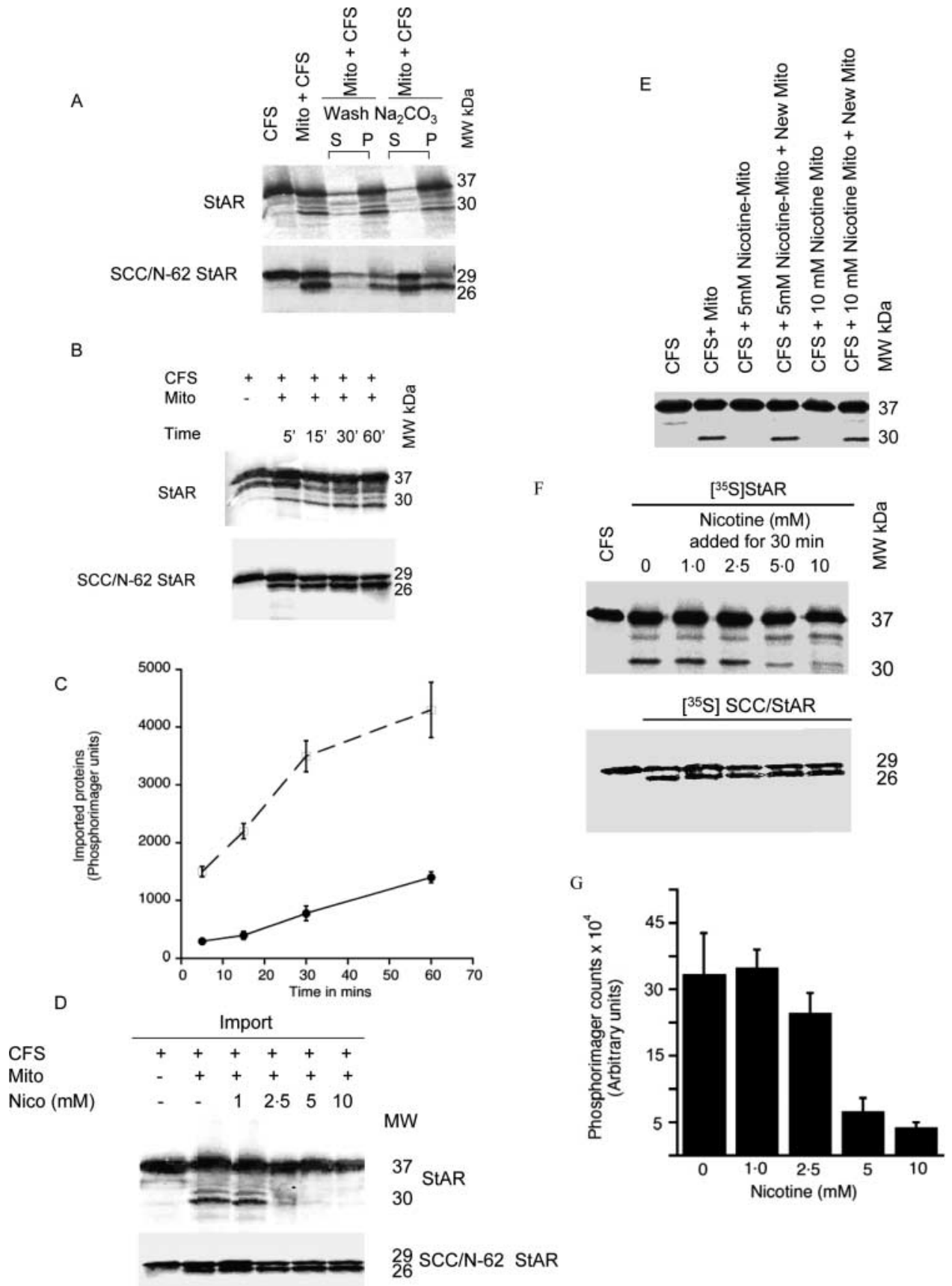

Figure 3 Mitochondrial StAR import. (A) Cell-free synthesised (CFS) ${ }^{35}$ S-StAR and SCC/N-62 StAR were imported into isolated mitochondria. (B) Import kinetics. StAR and SCC/N-62 StAR were imported kinetically from 5, 15, 30 and $60 \mathrm{~min}$. (C) Phosphorimager quantitation of the import efficiency of SCC/StAR and StAR. (D) Import of ${ }^{35} \mathrm{~S}-\mathrm{StAR}$ (top panel) and SCC/N-62 StAR (bottom panel) in the presence of $0,1,2 \cdot 5,5$ and $10 \mathrm{mM}$ nicotine. (E) Additional unexposed $100 \mu \mathrm{g}$ mitochondria was added to the incubation mixture of ${ }^{35} \mathrm{~S}-\mathrm{StAR}$ and mitochondria preincubated with the indicated concentrations of nicotine. (F) StAR and SCC/StAR import onto mitochondria preincubated with 0, 1, 2.5, 5 and $10 \mathrm{mM}$ nicotine. (G) Quantitative estimation of the imported $30 \mathrm{kDa}$ StAR (from (E), top section) and shows the means ( \pm S.E.M.) of three separate experiments. 
A

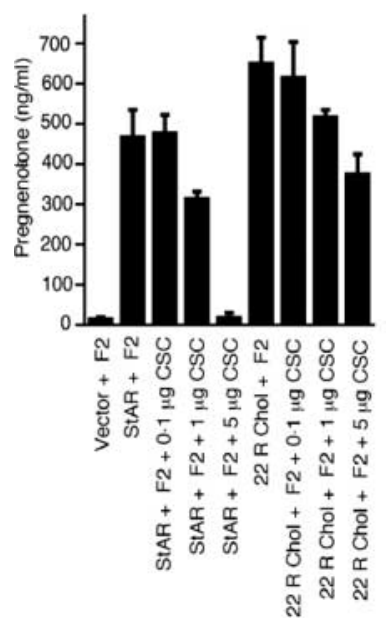

B

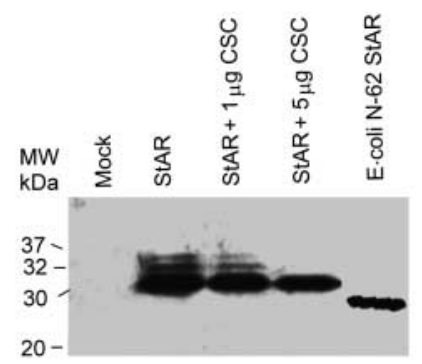

C

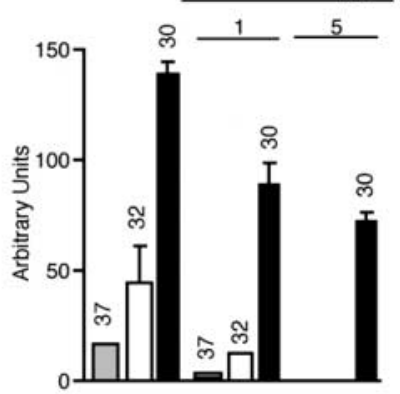

Figure 4 Pregnenolone synthesis in the presence of cigarette smoke condensate (CSC) transfected with StAR and F2. (A) COS- 1 cells preincubated with $0 \cdot 1,1 \cdot 0$ and $5 \mu \mathrm{g} / \mathrm{ml}$ of CSC. Data are the means ( \pm S.E.M.) of three separate experiments, each performed in triplicate. (B) Western blot of the

CSC-induced StAR transfected COS- 1 cells. The E. coli expressed N-62 StAR is a control present in the last lane. (C) Quantitation of the intensity of the 37, 32 and $30 \mathrm{kDa}$ bands from western blot and show the means ( \pm S.E.M.) of three separate experiments. The $Y$-axis is the intensity of the expressed protein and $\mathrm{X}$-axis is the concentrations of CSC.

maximum of the spectrum appears between 335 and $354 \mathrm{~nm}$ and reflects the compactness of a protein. In the absence of nicotine, biosynthetic N-62 StAR showed an emission maximum at $337 \cdot 2 \pm 0 \cdot 2 \mathrm{~nm}$, and in the presence of increasing concentrations of nicotine from 0.01 to $10 \mathrm{mM}$, the emission maximum remained unchanged (Fig. 6A), but the fluorescence intensity decreased, suggesting that nicotine possibly interacted with StAR. Therefore, we determined the binding of StAR with nicotine by measuring the change in free energy $(\Delta G)$ of StAR (Pace 1986, 1992). The unfolding of N-62 StAR (Fig. 6B) started at $1.75 \mathrm{M}$ urea with a transitional midpoint at $2.5 \mathrm{M}$ urea and completed melting at $4.0 \mathrm{M}$ urea. These curves fitted a two-state model where the free energy difference between the native $(\mathrm{N})$ and unfolded $(\mathrm{U})$

A

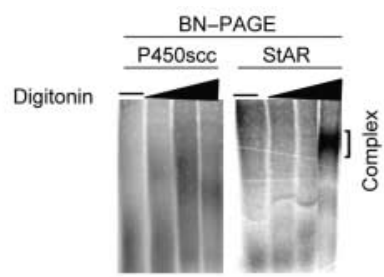

B

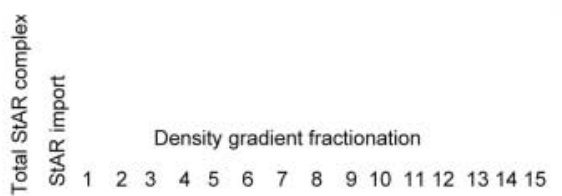

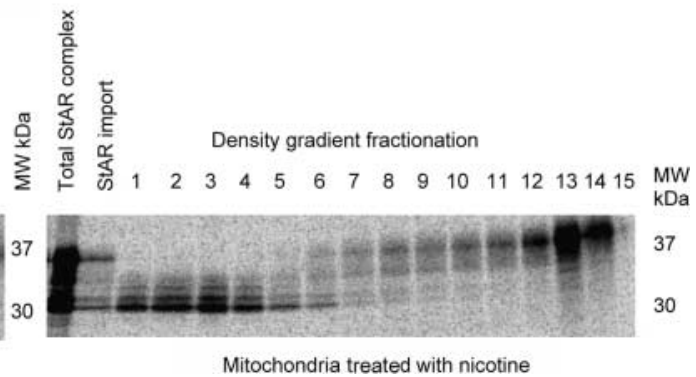

Figure 5 Effect of nicotine on the StAR-mitochondrial import complex. (A) Digitonin extraction of the ${ }^{35} \mathrm{~S}-\mathrm{StAR}$ and P450scc on the BN-PAGE. (B and C) Analysis of the StAR density gradient centrifugation by SDS-PAGE, where $B$ and $C$ represent mitochondria untreated and treated with nicotine respectively. 
A

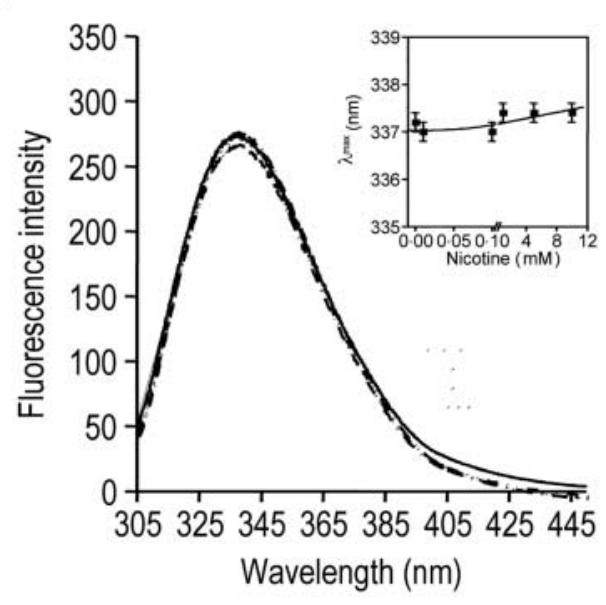

C

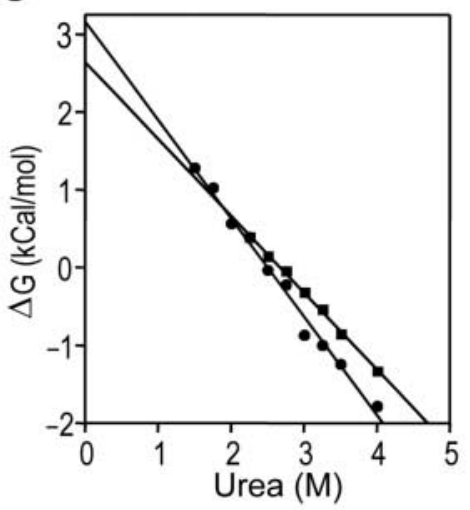

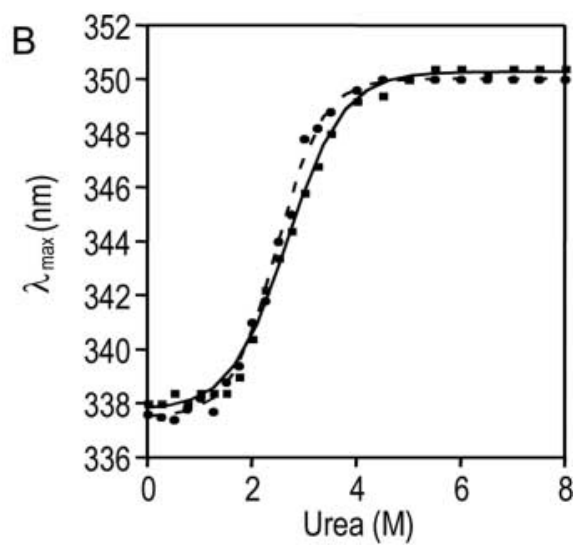

D

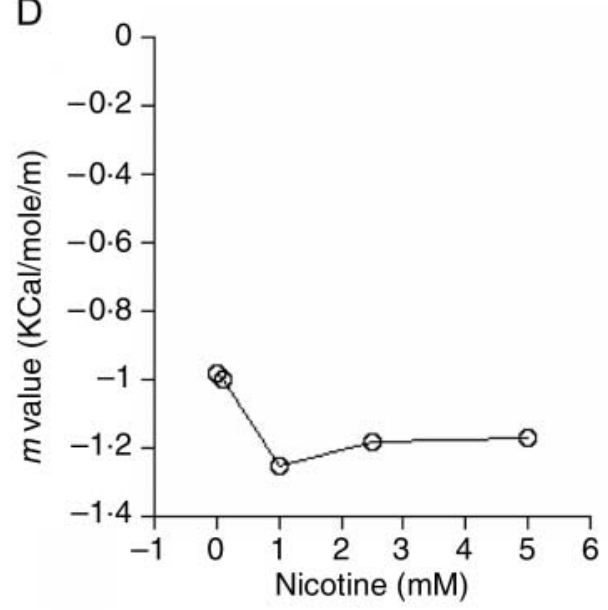

$\mathrm{E}$

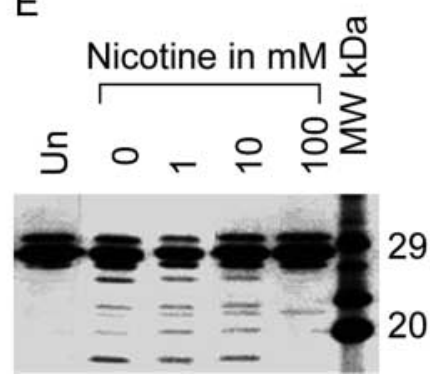

Figure 6 Effect of nicotine on N-62 StAR conformation. (A) Fluorescence spectra of N-62 StAR in the presence of $0 \cdot 1,0 \cdot 01,1,5$ and $10 \mathrm{mM}$ nicotine. The inset is the plot of emission maxima with different concentrations of nicotine. (B) Effect of nicotine on the stability of N-62 StAR in the absence (solid line) and presence of nicotine (broken line). (C) Determination of free energy of unfolding $(\Delta G)$ of N-62 StAR in the absence ( $\square$ ) and presence $(O)$ of nicotine. (D) A plot of the surface area $m$ value plotted against nicotine concentration. (E) Partial proteolysis of StAR in the presence of indicated concentrations of nicotine.

states depends on the urea concentration. Thus, $\Delta G=$ $\Delta G_{\mathrm{H} 2 \mathrm{O}}-m$ (Urea), where $\Delta G$ is the free-energy difference at any urea concentration. $\Delta G_{\mathrm{H} 2 \mathrm{O}}$ is the free energy difference in the absence of urea and $m$ is a solvent denaturant value that measures the rate of change of $\Delta G$ as a function of urea concentration
(Wrabl \& Shortle 1999); therefore the $m$ value is proportional to the new surface area of protein that becomes accessible upon unfolding (Wrabl \& Shortle 1999). The $\Delta G$ of unfolding of N-62 StAR in the presence and absence of nicotine was $3 \cdot 143$ and $2 \cdot 467 \mathrm{kcal} / \mathrm{mol}$ (Fig. $6 \mathrm{C}$ ), and the $m$ value changed 
from -0.87 to $-1.241 \mathrm{kcal} / \mathrm{mol}$ per $m$ with increasing nicotine concentrations (Fig. 6D), suggesting a partial stabilisation of StAR.

To determine the partial stabilisation of StAR in the presence of nicotine, we proteolysed StAR and analysed the protection of StAR domains in SDS-PAGE (Fig. 6E). Trypsin is a highly specific enzyme that digests after lysine and arginine residues at or above $\mathrm{pH} 7 \cdot 0$. Therefore, we incubated biosynthetic N-62 StAR in the presence of different concentrations of nicotine but with a fixed concentration of trypsin for a limited time at room temperature. To visualise the protolysed band by Coomassie blue dye, we incubated microgram quantities of biosynthetic N-62 StAR and with higher concentrations of nicotine during this proteolysis reactions. In the absence of nicotine or at lower concentrations of nicotine, the proteolytic pattern of $\mathrm{N}-62$ StAR was similar to our earlier findings (Bose $e t$ al. 1999), and at higher nicotine concentrations N-62 StAR became resistant to proteolysis (Fig. 6E, last lane), indicating that nicotine stabilised StAR's conformation.

\section{Discussion}

Steroidogenic activity and cholesterol transport are related to the stability and expression of StAR. This activity depends on the availability of the active protein at the OMM and only newly synthesised StAR can foster cholesterol (Artemenko et al. 2001). Western blotting of the nicotine-treated and -untreated cells with different antibodies confirmed that nicotine did not affect the outer mitochondrial and matrix proteins. Therefore, the mitochondrial general protein import pore (GIP; Neupert 1997) for protein import and sorting assembly remains unaffected. The import of StAR is reduced but not the signal sequence substituted StAR, so there is a StAR-specific transport mechanism through the OMM. Thus, the OMM-associated protein that StAR interacts with during its import and the transport of cholesterol, is the key factor affected by nicotine.

The view that StAR may act as an intracellular hormone fostered the idea that mitochondria might have specific receptor(s) that are required for StAR's action, other than those involved in protein import. When N-62 StAR is expressed in transfected cells, it transfers cholesterol to the peroximal and other membranes in addition to the OMM (Kallen et al. 1998), suggesting that the principal function of the mitochondrial leader is to target StAR exclusively to the OMM for its import. N-62 StAR equally transports cholesterol similar to full-length StAR to the mitochondrial matrix, and works in a partially open conformation. Therefore, the organisation of OMM proteins is crucial for mitochondrial function. This is especially true of mitochondrial targeted nuclearencoded proteins which may use preexisting
OMM-associated proteins as receptors or may associate with the OMM, as is the case with OMM-associated $\beta$-barrel proteins (Koehler 2004). Therefore, the import receptors at the OMM may be affected by nicotine (Hinsch et al. 2001). As StAR's conformation remained unchanged with increasing concentrations of nicotine, it is likely that nicotine affected the conformation of the cytoplasmic proteins associated with the OMM, thereby inhibiting StAR's interaction with the OMM.

The model structure suggested that StAR should work at the inner mitochondrial space to shuttle cholesterol into the matrix. StAR's co-crystal structure (Roderick et al. 2002) and the three-dimensional crystal structure of StARD4 (Romanowski et al. 2002) suggested that StAR must work at the cytoplasmic side of the OMM by partially opening its conformation. The absence of any significant difference in the CD spectrum between nicotine-treated and -untreated StAR indicated no interaction or minor interaction of StAR with nicotine. The small change in free energy $(-0.67 \mathrm{kcal} / \mathrm{mol})$ suggested that nicotine is associated with StAR in a molten globule state where StAR is in a partially open conformation due to the presence of more water molecules inside the protein core (Privalov 1996). The unimported StAR at the OMM was most likely proteolysed by the proteosomal proteases (Granot et al. 2003) or through ubiquitin-mediated proteases, which is a mandatory mechanism of the degradation of proteins for proper functioning of the cellular process (Lee et al. 2001). Thus, a lesser availability of StAR to transport adequate cholesterol into the mitochondria resulted in reduced pregnenolone.

Incubation of mitochondria with nicotine reduced StAR import, but not the import of SCC/N-62 StAR. The difference between StAR and SCC/StAR is the leader sequence, and both of them are imported into the mitochondrial matrix. Thus, there is a StARsequence leader-specific mechanism associated with StAR import into the mitochondria. All proteins must be imported through the mitochondrial import channel Tom40, where the outer mitochondrial Translocases (Tom) act as receptors for the incoming mitochondrial-targeted proteins for their further association with the OMM or their translocation and sorting through the IMM using the smaller Tim proteins. As $\beta$-barrel proteins like Tom 40 or porin (also known as VDAC) do not always use the preexisting Tom40 and VDAC channel for association with the OMM, it is possible that the StAR import process was hindered either with voltage dependent anion channel (VDAC) or with Tom40 after nicotine exposure. The minor import inhibition of SCC/N-62 StAR rules out the possibility of an alteration of the Tom 40 channel. Thus, we hypothesise that the most abundant OMMassociated protein, VDAC, is affected by nicotine. 
In summary, in this manuscript we have provided the following evidence: 1) StAR expression is reduced due to the presence of toxins like nicotine, 2) reduction of pregnenolone synthesis is due to the damage or blockage of the OMM, 3) StAR is stabilised by nicotine resulting in decreased interaction with the OMM, 4) temporary exposure to nicotine blocks both StAR and cholesterol transport, 5) the effect of cigarette smoke is stronger than that of nicotine alone and decreases the expression of protein at the OMM and 6) the import mechanism for StAR is different from other mitochondrial proteins imported into the same mitochondrial compartment.

\section{Acknowledgements}

The authors are thankful to Dr Arthur Edison for providing different instrumental facilities. Authors' contributions: $\mathrm{M} \mathrm{B}$ and $\mathrm{H} \mathrm{S} \mathrm{B}$ designed experiments, M B performed all the experiments and wrote the paper. M B is thankful to Kevin Fortin for reading the manuscript. D D recorded one fluorescence emission spectrum and Y C performed one western blot. H S B is thankful to Dr Walter L Miller for different antibodies used in this manuscript. The authors are also thankful to Drs Charles Wood and Maureen Keller-Wood for adrenal tissues and Vishwanath R Lingappa from UCSF for $\mathrm{Sp} 6$ vector. This work was supported by a grant from the James and Esther King Foundation, Florida Department of Health to H S B. The authors declare that there is no conflict of interest that would prejudice the impartiality of this scientific work.

\section{References}

Arakane F, Sugawara T, Nishino H, Liu Z, Holt JA, Pain D, Stocco DM, Miller WL \& Strauss JF, III. 1996 Steroidogenic acute regulatory protein (StAR) retains activity in the absence of its mitochondrial targeting sequence: Implications for the mechanism of StAR action. PNAS 93 13731-13736.

Artemenko IP, Zhao D, Hales DB, Hales KH \& Jefcoate CR 2001 Mitochondrial processing of newly synthesized steroidogenic acute regulatory protein (StAR), but not total StAR, mediates cholesterol transfer to cytochrome $\mathrm{P} 450$ side chain cleavage enzyme in adrenal cells. Journal of Biological Chemistry 276 46583-46596.

Aydos K, Guren M, Can B \& Ergun A 2001 Nicotine toxicity to the ultrastructure of the testis in rats. BJU International 88 622-626.

Banzet N, Francois D \& Polla B 1999 Tobacco smoke induces mitochondrial depolarization along with cell death: effects of antioxidants. Redox Report 4 229-236.

Benowitz NL 1996 Cotinine as a biomarker of environmental tobacco smoke exposure. Epidemiologic Reviews 18 188-204.

Blake CA 1974 Localization of the inhibitory actions of ovulationblocking drugs on release of luteinizing hormone in ovariectomized rats. Endocrinology 95 999-1004

Blake CA 1978 Paradoxical effects of drugs acting on the central nervous system on the preovulatory release of pituitary luteinizing hormone in pro-oestrous rats. Journal of Endocrinology 79 319-326.
Blake CA, CScramuzzi RJ, Norman RL, Kanematsu S \& Sawyer CH 1972 Effect of nicotine on the proestrous ovulatory surge of LH in the rat. Endocrinology 91 1253-1258.

Bose HS, Sugawara T, Strauss JF, III. \& Miller WL 1996 The pathophysiology and genetics of congenital lipoid adrenal hyperplasia. New England Journal of Medicine 335 1870-1878.

Bose HS, Baldwin MA \& Miller WL 1998 Incorrect folding of steroidogenic acute regulatory protein (StAR) in congenital lipoid adrenal hyperplasia. Biochemistry 37 9768-9775.

Bose HS, Whittal RM, Baldwin MA \& Miller WL 1999 The active form of the steroidogenic acute regulatory protein, StAR, appears to be a molten globule. PNAS 96 7250-7255.

Bose HS, Sato S, Aisenberg J, Shalev SA, Matsuo N \& Miller WL 2000 Mutations in the steroidogenic acute regulatory protein (StAR) in six patients with congenital lipoid adrenal hyperplasia. Journal of Clinical Endocrinology and Metabolism 85 3636-3639.

Bose HS, Lingappa VR \& Miller WL 2002 Rapid regulation of steroidogenesis by mitochondrial protein import. Nature 417 87-91.

Brentano ST, Black SM, Lin D \& Miller WL 1992 cAMP posttranscriptionally diminishes the abundance of adrenodoxin reductase mRNA. PNAS 89 4099-4103.

Calafat AM, Polzin GM, Saylor J, L AD \& Watson CH 2004 Determination of tar, nicotine, and carbon monoxide yields in the mainstream smoke of selected international cigarettes. Tobacco Control 13 45-51.

Granot Z, Geiss-Friedlander R, Melamed-Brook N, Eimerl S, Timberg R, Weiss AM, Hales KH, Hales DB, Stocco DM \& Orly J 2003 Proteolysis of normal and mutated steroidogenic acute regulatory proteins in the mitochondria; the fate of unwanted proteins. Molecular Endocrinology 17 2461-2476.

Hakki A, Friedman H \& Pross S 2001 Nicotine inhibition of apoptosis in murine immune cells. Experimental Biology and Medicine 226 947-953.

Harikrishna JA, Black SM, Szklarz GD \& Miller WL 1993 Construction and function of fusion enzymes of the human cytochrome P450scc system. DNA and Cell Biology 12 371-379.

Hinsch KD, Elvira A \& Konrad L 2001 VDAC2 (porin-2) expression pattern and localization in the bovine testis. Biochimica et Biophysica Acta 1518 329-333.

Hsu HJ, Liang MR, Chen CT \& Chung BC 2006 Pregnenolone stabilizes microtubules and promotes zebrafish embryonic cell movement. Nature 439 480-483.

Kallen CB, Billheimer JT, Summers SA, Stayrook SE, Lewis M \& Strauss JF, III. 1998 Steroidogenic acute regulatory protein (StAR) is a sterol transfer protein. Journal of Biological Chemistry 273 26285-26288.

Koehler CM 2004 New developments in mitochondrial assembly. Annual Review of Cell and Developmental Biology 20 309-335.

Lee C, Schwartz MP, Prakash S, Iwakura M \& Matouschek A 2001 ATPdependent proteases degrade their substrates by processively unraveling them from the degradation signal. Molecular Cell 7 627-637.

Li JM \& Shore GC 1992 Reversal of the orientation of an integral protein of the mitochondrial outer membrane. Science $\mathbf{2 5 6}$ 1815-1817.

Lin D, Sugawara T, Strauss JF, III., Clark BJ, Stocco DM, Saenger P, Rogol A \& Miller WL 1995 Role of steroidogenic acute regulatory protein in adrenal and gonadal steroidogenesis. Science 267 1828-1831.

Miller WL 1988 Molecular biology of steroid hormone synthesis. Endocrine Reviews 9 295-318.

Mlynarcikova A, Fickova M \& Scsukova S 2005 Ovarian intrafollicular processes as a target for cigarette smoke components and selected environmental reproductive disruptors. Endocrine Regulations 39 $20-31$.

Nakae J, Tajima T, Sugawara T, Arakane F, Hanaki K, Hotsubo T, Igarashi N, Igarashi Y, Ishii T, Koda N et al. 1997 Analysis of the steroidogenic acute regulatory protein $(S t A R)$ gene in Japanese patients with congenital lipoid adrenal hyperplasia. Human Molecular Genetics 6 571-576. 
Neupert W 1997 Protein import into mitochondria. Annual Review of Biochemistry 66 863-917.

Pace CN 1986 Determination and analysis of urea and guanidine hydrochloride denaturation curves. Methods in Enzymology 131 266-280.

Pace CN 1992 Contribution of the hydrophobic effect to globular protein stability. Journal of Molecular Biology 226 29-35.

Patil S, Patil S, Bhaktaraj B \& Patil SB 1999 Effect of graded doses of nicotine on ovarian and uterine activities in albino rats. Indian Journal of Experimental Biology 37 184-186.

Pillsbury HC \& Bright CC 1972 Comparison of aliquot and complete sample procedure for the determination of nicotine in cigarette smoke. Journal of the Association of Official Analytical Chemists55 636-638.

Privalov PL 1996 Intermediate states in protein folding. Journal of Molecular Biology 258 707-725.

RJ Reynolds Tobacco Co. 1988 Chemical and Biological Studies on New Cigarette Prototypes that Heat Instead of Burn Tobacco, Winston-Salem, NC: RJ Reynolds Company.

Roderick SL, Chan WW, Agate DS, Olsen CR, Vetting MW, Rajashankar KR \& Cohen DE 2002 Structure of human phosphatidylcholine transfer protein in complex with its ligand. Nature Structural Biology 9 507-511.

Romanowski MJ, Soccio RE, Breslow JL \& Burley SK 2002 Crystal structure of the Mus musculus cholesterol-regulated START protein 4 (StarD4) containing a StAR-related lipid transfer domain. PNAS 99 6949-6954.

Sarasin A, Schlumpf M, Muller M, Fleischmann I, Lauber ME \& Lichtensteiger W 2003 Adrenal-mediated rather than direct effects of nicotine as a basis of altered sex steroid synthesis in fetal and neonatal rat. Reproductive Toxicology 17 153-162.

Schagger H \& von Jagow G 1993 Blue native electrophoresis for isolation of membrane protein complexes in enzymatically active form. Analytical Biochemistry 199 223-231.

Schagger H, Cramer WA \& von Jagow G 1994 Analysis of molecular masses and oligomeric states of protein complexes by blue native electrophoresis and isolation of membrane protein complexes by two dimensional native electrophoresis. Analytical Biochemistry 217 220-230.
Schwartz MP \& Matouschek A 1999 The dimensions of the protein import channels in the outer and inner mitochondrial membrane. PNAS 96 13086-13090.

Shihadeh A \& Saleh R 2005 Polycyclic aromatic hydrocarbons, carbonmonoxide, 'tar', and nicotine in the mainstream smoke aerosol of the narghile water pipe. Food and Chemical Toxicology 43 655-661.

Stocco DM \& Clark BJ 1996 Regulation of the acute production of steroids in steroidogenic cells. Endocrine Reviews 17 221-244.

Sugawara T, Holt JA, Driscoll D, Strauss JF, III., Lin D, Miller WL, Patterson D, Clancy KP, Hart IM, Clark BJ et al. 1995 Human steroidogenic acute regulatory protein (StAR): functional activity in COS-1 cells, tissue-specific expression, and mapping of the structural gene to $8 \mathrm{p} 11.2$ and an expressed pseudogene to chromosome 13. PNAS 92 4778-4782.

Tapper AR, McKinney SL, Nashmi R, Schwarz J, Deshpande P, Labarca C, Whiteaker P, Marks MJ, Collins AC \& Lester H 2004 Nicotine activation of $\alpha 4^{*}$ receptors: sufficient for reward, tolerance, and sensitization. Science 306 1029-1032.

Weisberg E 1985 Smoking and reproductive health. Clinical Reproduction and Fertility 3 175-186.

Wiedemann N, Kozak V, Chacinska A, Schonfisch B, Rosepert S, Ryan MT, Pfanner N \& Meisinger C 2003 Machinery for protein sorting and assembly in the outer mitochondrial membrane. Nature 424 565-571.

Wrabl J \& Shortle D 1999 A model of the changes in denatured state structure underlying $m$ value effects in staphyloccal nuclease. Nature Structural Biology 6 876-883.

Xie Y-X, Bezard E \& Zhao B-L 2005 Investigating the receptorindependent neuroprotective mechanisms of nicotine in mitochondria. Journal of Biological Chemistry 250 32405-32412.

Received in final form 5 May 2007

Accepted 15 May 2007

Made available online as an Accepted Preprint 21 May 2007 